\title{
Markov partitions for hyperbolic sets
}

\author{
Todd Fisher and Himal Rathnakumara
}

(Communicated by Kenneth S. Berenhaut)

\begin{abstract}
We show that if $f$ is a diffeomorphism of a manifold to itself, $\Lambda$ is a mixing (or transitive) hyperbolic set, and $V$ is a neighborhood of $\Lambda$, then there exists a mixing (or transitive) hyperbolic set $\tilde{\Lambda}$ with a Markov partition such that $\Lambda \subset$ $\tilde{\Lambda} \subset V$. We also show that in the topologically mixing case the set $\tilde{\Lambda}$ will have a unique measure of maximal entropy.
\end{abstract}

\section{Introduction}

A dynamical system consists of a space and a rule to dictate the evolution of the points in the space. In particular, a discrete dynamical system $(X, f)$ consists of a topological space $X$ and a map $f: X \rightarrow X$. The $n$th iterate of $f$, denoted $f^{n}$, is defined as the map $f$ composed $n$ times, where $n \in \mathbb{N}$. If $f$ is a bijection, then its inverse $f^{-1}$ exists and we can form the $n$th iterate of $f^{-1}$ by composition, $f^{-n}: X \rightarrow X$.

We assume in this paper that the maps associated with dynamical systems are homeomorphisms so that $f^{-1}$ exists and $f^{-n}$ is well-defined. In the study of dynamical systems it is important to look at the overall effect of the rule for individual points in the space. In this analysis we look at orbits of points in the space where the orbit of a point $x \in X$ is defined as

$$
\mathcal{O}(x)=\left\{f^{n}(x) \in X: n \in \mathbb{Z}\right\} .
$$

Throughout the paper we let $M$ be a compact, smooth, boundaryless manifold and denote the set of diffeomorphisms from $M$ to itself by $\operatorname{Diff}(M)$. A set $X$ is invariant under $f$ if $f(X)=X$. Invariant sets play an important role in dynamical systems and often allow one to decompose a space into invariant "indecomposable" sets. A compact set $\Lambda \subset M$ that is invariant under $f \in \operatorname{Diff}(M)$ is a hyperbolic set

MSC2000: 37A35, 37D05, 37D15.

Keywords: Markov partitions, hyperbolic, entropy, specification, finitely presented. 
if there exists a splitting of the tangent space $T_{\Lambda} f=\mathbb{E}^{u} \oplus \mathbb{E}^{s}$ and positive constants $C \geq 1$ and $\lambda<1$ such that for any point $x \in \Lambda$ and any $n \in \mathbb{N}$ we have

$$
\begin{aligned}
\left\|D f_{x}^{n} v\right\| \leq C \lambda^{n}\|v\| & \text { for } v \in E_{x}^{s}, \\
\left\|D f_{x}^{-n} v\right\| \leq C \lambda^{n}\|v\| & \text { for } v \in E_{x}^{u} .
\end{aligned}
$$

Hyperbolic sets were introduced by Smale and Anosov in the 1960s. The compactness of the manifold together with the expansion and contraction in the tangent bundle allows for complicated and interesting orbit structures. Additionally, hyperbolic sets are structurally stable, or in other words, the dynamics of a hyperbolic set are preserved under perturbations.

One of the main tools in studying hyperbolic sets is the use of a Markov partition introduced by Adler and Weiss for hyperbolic toral automorphisms of the 2-torus [Adler and Weiss 1967]. Markov partitions are defined in Section 2. It was shown in [Fisher 2006] that if $f \in \operatorname{Diff}(M), \Lambda$ is a hyperbolic set for $f$, and $V$ is a neighborhood of $f$, then there exists a hyperbolic set $\tilde{\Lambda}$ for $f$ such that $\Lambda \subset \tilde{\Lambda} \subset V$ and $\tilde{\Lambda}$ has a Markov partition. For a Markov partition there is a canonically associated symbolic space called a subshift of finite type. (For the definition of a subshift of finite type see Section 2.)

Often one is interested in studying hyperbolic sets that satisfy additional properties. Two such properties are topological mixing and transitivity. A dynamical system $(X, f)$ is topologically mixing if for any open sets $U$ and $V$ there exists some $N \in \mathbb{N}$ such that $f^{n}(U) \cap V \neq \varnothing$ for all $n \geq N$. A dynamical system $(X, f)$ is transitive if there exists a point $x \in X$ such that the forward orbit of $x$,

$$
\mathcal{O}^{+}(x)=\left\{f^{n}(x): n \in \mathbb{N}\right\},
$$

is dense in $X$. A standard result about transitivity is the following: if $X$ is a locally compact Hausdorff space, then $(X, f)$ is topologically transitive if and only if for any open sets $U$ and $V$ in $X$ there exists some $n \in \mathbb{N}$ such that $f^{n}(U) \cap V \neq \varnothing$ [Brin and Stuck 2002, page 31].

The main result of the present work is that we can strengthen the result on Markov partitions in [Fisher 2006] with respect to topological mixing and transitivity.

Theorem 1.1. If $\Lambda$ is a topologically mixing hyperbolic set for $f \in \operatorname{Diff}(M)$ and $V$ is a neighborhood of $\Lambda$, then there exists a hyperbolic set $\tilde{\Lambda}$ for $f$ containing $\Lambda$ and contained in $V$ such that $(\tilde{\Lambda}, f)$ has a Markov partition coming from an associated mixing subshift of finite type. Furthermore, if $\Lambda$ is transitive, then $(\tilde{\Lambda}, f)$ has a Markov partition coming from an associated transitive subshift of finite type.

We note that a standard result is that if the subshift of finite type is mixing (or transitive) and associated to a Markov partition for a hyperbolic set, then the 
hyperbolic set is mixing (or transitive). Bowen [1974] provided a nice connection between mixing hyperbolic sets and the entropy for the system. The topological entropy of a dynamical system, denoted $h_{\text {top }}(f)$, is a number that, in a certain manner, measures the topological complexity of the system. Whereas, the measure theoretic entropy, denoted $h_{\mu}(f)$, of a dynamical system is a number that, in some manner, measures the complexity of the system as seen by the measure $\mu$.

A measure $\mu$ is invariant for the dynamical system $(X, f)$ if

$$
\mu\left(f^{-1}(A)\right)=\mu(A)
$$

for all measurable sets $A$. We denote the set of invariant Borel probability measures as $\mathcal{M}(f)$. If $X$ is a compact metrizable space and $f$ is continuous, then we know that $M(f) \neq \varnothing$ [Katok and Hasselblatt 1995, page 135]. The variational principle says that if $f$ is a homeomorphism of a compact metrizable space, then $h_{\text {top }}(f)=$ $\sup _{\mu \in \mathcal{M}(f)} h_{\mu}(f)$ [Katok and Hasselblatt 1995, page 181]. A measure $\mu \in \mathcal{M}(f)$ such that $h_{\text {top }}(f)=h_{\mu}(f)$ is a measure of maximal entropy. If there is a unique measure of maximal entropy, then $f$ is called intrinsically ergodic. From Theorem 1.1 and Bowen's results we are then able to show the following.

Corollary 1.2. If $\Lambda$ is a topologically mixing hyperbolic set and $V$ is a neighborhood of $\Lambda$, then there exists a hyperbolic set $\tilde{\Lambda}$ containing $\Lambda$ and contained in $V$ such that $\tilde{\Lambda}$ is intrinsically ergodic with respect to $f$.

\section{Background}

As we will be looking at subshifts of finite type we first review some definitions and facts about subshifts of finite type. Let $A=\left[a_{i j}\right]$ be an $n \times n$ matrix with entries of zeros and ones such that there is one or more one in each row and column. Such a matrix is called an adjacency matrix. Let $\mathscr{A}_{n}=\{1, \ldots, n\}$ and call a transition from $i$ to $j$ to be admissible for $A$ if $a_{i j}=1$. Define

$$
\Sigma_{A}=\left\{\omega=\left(\omega_{k}\right)_{k \in \mathbb{Z}} \mid \omega_{k} \in \mathscr{A}_{n} \text { and } \omega_{k} \omega_{k+1} \text { is admissible for all } k \in \mathbb{Z}\right\} .
$$

The map on $\Sigma_{A}$ defined by $\sigma(\omega)=\omega^{\prime}$ where $\omega_{j}^{\prime}=\omega_{j+1}$ is called the shift map. The subshift of finite type is the space $\left(\Sigma_{A}, \sigma\right)$ together with the product metric on $\Sigma_{A}$. A matrix $A$ is positive if each entry is positive. A matrix $A$ is primitive if there is some power $N \in \mathbb{N}$ such that $A^{N}$ is positive.

If a matrix $A$ is primitive, then the subshift of finite type associated with $A$ is topologically mixing. Furthermore, a subshift of finite type associated with an $M \times M$ matrix $A$ is transitive if and only if for each $i, j(1 \leq i, j \leq M)$ there exists some $n \in \mathbb{N}$ such that $a_{i j}^{n}>0$ [Robinson 1999, page 80]. 
A topological semiconjugacy between a pair of dynamical systems $(X, f)$ and $(Y, g)$ exists if there is a continuous surjective map $h: X \rightarrow Y$ such that

$$
h \circ f=g \circ h .
$$

The space $(Y, g)$ is called a factor of $(X, f)$, and $(X, f)$ is called an extension of $(Y, g)$.

A dynamical system $(X, f)$ where $X$ is a compact metric space and $f$ is a homeomorphism is expansive if there exists a constant $c>0$ such that for all $x, y \in X$ if $d\left(f^{n}(x), f^{n}(y)\right)<c$ for all $n \in \mathbb{Z}$, then $x=y$.

We now review some facts about expansive and finitely presented dynamical systems. For $\varepsilon>0$ and $x \in X$ the $\varepsilon$-stable set is

$$
W_{\varepsilon}^{s}(x)=\left\{y \in X \mid d\left(f^{n}(x), f^{n}(y)\right)<\varepsilon \text { for all } n \geq 0\right\},
$$

and the $\varepsilon$-unstable set is

$$
W_{\varepsilon}^{u}(x)=\left\{y \in X \mid d\left(f^{-n}(x), f^{-n}(y)\right)<\varepsilon \text { for all } n \geq 0\right\} .
$$

For $x \in X$ and $f: X \rightarrow X$, an expansive homeomorphism, the stable set is

$$
W^{s}(x)=\left\{y \in X \mid \lim _{n \rightarrow \infty} d\left(f^{n}(x), f^{n}(y)\right)=0\right\}
$$

and the unstable set is

$$
W^{u}(x)=\left\{y \in X \mid \lim _{n \rightarrow \infty} d\left(f^{-n}(x), f^{-n}(y)\right)=0\right\} .
$$

Let $(Y, f)$ be expansive and fix $\varepsilon<c / 2$, where $c$ is an expansive constant of $(Y, f)$. Following [Fried 1987] we define

$$
D_{\varepsilon}=\left\{(x, y) \in Y \times Y \mid W_{\varepsilon}^{s}(x) \text { meets } W_{\varepsilon}^{u}(y)\right\}
$$

and $[\cdot, \cdot]: D_{\varepsilon} \rightarrow Y$ so that $[x, y]=W_{\varepsilon}^{s}(x) \cap W_{\varepsilon}^{u}(y)$. It follows that $[\cdot, \cdot]$ is continuous.

Definition 2.1. A rectangle is a closed set $R \subset Y$ such that $R \times R \subset D_{\varepsilon}$.

For $R$ a rectangle and $x \in R$, denote the stable and unstable sets of $x$ in $R$, respectively, as

$$
W^{s}(x, R)=R \cap W_{\varepsilon}^{s}(x), \quad W^{u}(x, R)=R \cap W_{\varepsilon}^{u}(x) .
$$

A rectangle $R$ is proper if $R=\overline{\grave{R}}$, where $\stackrel{R}{ }$ denotes the interior of $R$.

Definition 2.2. Let $(Y, f)$ be expansive with constant $c>0$ and $0<\varepsilon<c / 2$. A finite cover $\mathscr{R}$ of $Y$ by proper rectangles with diameter $(R)<\varepsilon$ for any $R \in \mathscr{R}$ is a Markov partition if $R_{i}, R_{j} \in \mathscr{R}, x \in \stackrel{\circ}{R}_{i}$, and $f(x) \in \stackrel{\circ}{R}_{j}$, then $f\left(W^{s}\left(x, R_{i}\right)\right) \subset R_{j}, \quad f^{-1}\left(W^{u}\left(f(x), R_{j}\right)\right) \subset R_{i}, \quad$ and $\quad \stackrel{\circ}{R}_{i} \cap \stackrel{\circ}{R}_{j}=\varnothing$ if $i \neq j$. 
For a Markov partition $\mathscr{R}$ of a system $(X, f)$ we define the adjacency matrix $A$ such that $a_{i j}=1$ if $f\left(\stackrel{\circ}{R}_{i}\right) \cap \stackrel{\circ}{R}_{j} \neq \varnothing$. The subshift of finite type $\left(\Sigma_{A}, \sigma\right)$ is said to be associated with $\mathscr{R}$ and there is a canonical semiconjugacy $h$ from $\left(\Sigma_{A}, \sigma\right)$ to $(X, f)$.

Fried [1987] defined finitely presented systems as expansive homeomorphisms of a compact space that are factors of a subshift of finite type. In the same paper he shows that any finitely presented dynamical system has a Markov partition.

Remark 2.3. For $f \in \operatorname{Diff}(M)$ and $\Lambda$ a hyperbolic set for $f$, the system $\left(\Lambda,\left.f\right|_{\Lambda}\right)$ is expansive. Furthermore, any subshift of finite type is expansive. Also, for a hyperbolic set $\Lambda$ for a diffeomorphism and $x \in \Lambda$, the sets $W^{s}(x)$ and $W^{u}(x)$ are injectively immersed submanifolds of Euclidean spaces.

\section{Results}

Proof of Theorem 1.1. Before proceeding to the proof of Theorem 1.1 we first review some facts about shadowing for hyperbolic sets. A sequence $\left\{x_{k}\right\}_{a}^{b}$ is an $\varepsilon$ chain if $d\left(f\left(x_{k}\right), x_{k+1}\right)<\varepsilon$ for all $k$ where $-\infty \leq a<b \leq \infty$. A point $y \delta$-shadows an $\varepsilon$-chain $\left\{x_{k}\right\}$ if $d\left(f^{k}(y), x_{k}\right)<\delta$ for all $k$. We next state the Shadowing Theorem [Brin and Stuck 2002, page 113].

Theorem 3.1 (Shadowing Theorem). Let $M$ be a Riemannian manifold, $d$ the natural distance function, $f$ a diffeomorphism of $M$ to itself, and $\Lambda$ a hyperbolic set for $f$. Then for every $\delta>0$ there exists an $\varepsilon>0$ such that if $\left\{x_{n}\right\}$ is an $\varepsilon$-chain of $f$ and $d\left(x_{k}, \Lambda\right)<\varepsilon$ for all $k$, then there is some $y \in \bigcup_{x \in \Lambda} B_{\varepsilon}(x)$ that $\delta$-shadows the $\varepsilon$-chain $\left\{x_{k}\right\}$.

Proof of Theorem 1.1. We first assume that $\Lambda$ is topologically mixing. To prove the theorem it will be sufficient to show that the subshift of finite type constructed in [Fisher 2006] giving the hyperbolic set $\tilde{\Lambda}$ will be topologically mixing.

Let $U$ be a neighborhood of $\Lambda$. A standard result for hyperbolic sets states that there is a neighborhood $V$ of $\Lambda$ such that $\bar{V} \subset U$ and $\Lambda_{V}=\bigcap_{n \in \mathbb{Z}} f^{n}(\bar{V})$ is hyperbolic [Katok and Hasselblatt 1995, page 271]. Let $d(\cdot, \cdot)$ be an adapted metric on $\Lambda_{V}$. Note that this can be extended continuously to a neighborhood $V^{\prime} \subset U$ of $\Lambda_{V}$.

Fix $\eta>0$ and $\delta \leq \eta$ such that for any two points $x, y \in \Lambda_{V}$, if $d(x, y)<\delta$ then

$$
f^{-1}\left(W_{\eta}^{s}(f(x))\right) \cap f\left(W_{\eta}^{u}\left(f^{-1}(y)\right)\right)=W_{\eta}^{s}(x) \cap W_{\eta}^{u}(y)
$$

consists of one point, and the set $\bigcup_{x \in \Lambda} B_{2 \eta}(x)$ is contained in $V \cap V^{\prime}$; see [Fisher 2006] for an argument explaining the existence of $\eta$ and $\delta$. Fix $0<\varepsilon \leq \delta / 2$ as in the conclusion of the Shadowing Theorem so that every $\varepsilon$-orbit is $\delta / 2$-shadowed and contained in $V \cap V^{\prime}$. 
Let $v<\varepsilon / 2$ such that $d(f(x), f(y))<\varepsilon / 2$ and $d\left(f^{-1}(x), f^{-1}(y)\right)<\varepsilon / 2$ when $d(x, y)<v$ for any $x, y \in \Lambda_{V}$. Let $\left\{p_{i}\right\}_{i=1}^{N}$ be a $\nu$-dense set of points in $\Lambda$ and let the adjacency matrix $A$ be defined by

$$
a_{i j}=\left\{\begin{array}{l}
1 \text { if } d\left(f\left(p_{i}\right), p_{j}\right)<\varepsilon, \\
0 \text { if } d\left(f\left(p_{i}\right), p_{j}\right) \geq \varepsilon .
\end{array}\right.
$$

Let $\left(\Sigma_{A}, \sigma\right)$ be the subshift of finite type associated with $A$. Then we know there exists a hyperbolic set $\tilde{\Lambda}$ contained in $\bar{V}$ [Fisher 2006] such that $\tilde{\Lambda} \subset \Lambda_{V}$, that contains $\Lambda$ and there exists a semiconjugacy $\beta: \Sigma_{A} \rightarrow \tilde{\Lambda}$. To see that $\tilde{\Lambda}$ is topologically mixing it is sufficient to see that $\Sigma_{A}$ is topologically mixing.

We now show that $\Sigma_{A}$ is topologically mixing by showing that $A$ is primitive. Given sets $B_{v}\left(p_{i}\right)$ and $B_{v}\left(p_{j}\right)$ there exists some $N_{i j}$ such that for all $n \geq N_{i j}$ we have

$$
f^{n}\left(B_{v}\left(p_{i}\right)\right) \cap B_{v}\left(p_{j}\right) \neq \varnothing,
$$

since $\Lambda$ is topologically mixing for $f$. We let $M=\max \left\{N_{i j}\right\}$. Then

$$
f^{n}\left(B_{v}\left(p_{i}\right)\right) \cap B_{v}\left(p_{j}\right) \neq \varnothing
$$

for all $n \geq M$. We now show that this implies that $a_{i j}^{n}>0$ for all $n \geq M$. This is equivalent to showing there is a sequence of $(n+1)$-symbols coming from $\mathscr{A}_{N}$ such that each transition is allowed and the sequence starts with $i$ and ends with $j$ [Robinson 1999, page 76].

Indeed, let $n \geq M$ and $x \in f^{n}\left(B_{v}\left(p_{i}\right)\right) \cap B_{v}\left(p_{j}\right)$. Since

$$
\bigcup_{k=1}^{N} f\left(B_{v}\left(p_{k}\right)\right)=\Lambda,
$$

we know that there exists some $p_{i_{1}}$ such that $x \in f\left(B_{v}\left(p_{i_{1}}\right)\right)$. By the definition of $v$ we know that $d\left(f\left(p_{i_{1}}\right), p_{j}\right)<\varepsilon$ and $i_{1}$ to $j$ is an allowed transition in $\Sigma_{A}$. Inductively, let $1 \leq k \leq n-2$ and assume that for each $l$ such that $1 \leq l \leq k$ there is some $p_{i_{l}}$ such that $f^{-l}(x) \in f\left(B_{v}\left(p_{i_{l}}\right)\right)$ and

$$
\begin{aligned}
d\left(f\left(p_{i_{l}}\right), p_{j}\right)<\varepsilon & \text { if } l=1, \\
d\left(f\left(p_{i_{l}}\right), p_{i_{l-1}}\right)<\varepsilon & \text { else. }
\end{aligned}
$$

Then $i_{k} i_{k-1} \cdots i_{1} j$ is a sequence of $k+1$ symbols in $\mathscr{A}_{N}$ with allowed transitions and $f^{-l}(x) \in B_{v}\left(p_{i_{l}}\right)$ for all $1 \leq l \leq k$. We know that $f^{-(k+1)}(x) \in f\left(B_{v}\left(p_{i_{k+1}}\right)\right)$ for some $i_{k+1} \in \mathscr{A}_{N}$ and

$$
d\left(f\left(p_{i_{k+1}}\right), p_{i_{k}}\right)<\varepsilon .
$$

Hence, $i_{k+1}$ to $i_{k}$ is an allowed transition in $\Sigma_{A}$ and $i_{k+1} i_{k} \cdots i_{1} j$ is a sequence of $k+2$ symbols in $\mathscr{A}_{N}$ with allowed transitions. Therefore, there is a sequence 
$i_{n-1} i_{n-2} \cdots i_{1} j$ of $n$-terms in $\mathscr{A}_{N}$ with allowed transitions. Finally, we know that $f^{-n}(x) \in B_{v}\left(p_{i}\right)$ and

$$
f^{-(n-1)}(x) \in f\left(B_{v}\left(p_{i}\right)\right) \cap B_{v}\left(p_{i_{n-1}}\right) .
$$

So $i$ to $i_{n-1}$ is an allowed transition. Hence, $i i_{n-1} \cdots i_{1} j$ is an allowed word in $\Sigma$ and $a_{i j}^{n}>0$. Therefore, $A$ is primitive and $\Sigma_{A}$ is topologically mixing.

The proof of the transitive case is similar. Indeed, given sets $B_{v}\left(p_{i}\right)$ and $B_{v}\left(p_{j}\right)$ there exists some $N_{i j}$ such that

$$
f^{N_{i j}}\left(B_{v}\left(p_{i}\right)\right) \cap B_{v}\left(p_{j}\right) \neq \varnothing .
$$

Hence, a similar argument as above shows that $a_{i j}^{N_{i j}}>0$ and $\Sigma_{A}$ is transitive.

Intrinsic ergodicity for mixing hyperbolic sets. The proof of Corollary 1.2 will use the property of specification. A specification, $S=(\tau, P)$, for a dynamical system consists of

(1) a finite collection $\tau=\left\{I_{1}, \ldots, I_{n}\right\}$ of finite intervals $I_{i}=\left[a_{i}, b_{i}\right] \subset \mathbb{Z}$, and

(2) a map $P: \bigcup_{i=1}^{m} I_{i} \longrightarrow X$ such that $f^{t_{2}-t_{1}}\left(P\left(t_{1}\right)\right)=P\left(t_{2}\right)$. for all $t_{1}, t_{2} \in I_{i} \in \tau$.

A specification $S$ is said to be $r$-spaced, where $r \in \mathbb{N}$, if $a_{i+1}>b_{i}+r$ for all $i \in\{1, \ldots, n-1\}$ and the minimal such $r$ is called the spacing of $S$. A specification $S=(\tau, P)$ provides a way of parametrizing a collection of orbit segments $\tau$ of $f$. We say that $S$ is $\varepsilon$-shadowed by $x \in X$ if $d\left(f^{n}(x), P(n)\right)<\varepsilon$ for all $n \in \bigcup_{i=1}^{m} I_{i}$.

Definition 3.2. Let $X$ be a compact metric space and $f: X \rightarrow X$ a homeomorphism. The dynamical system $(X, f)$ is said to have the specification property if for all $\varepsilon>0$ there exists an $M_{\varepsilon} \in N$ such that any $M_{\varepsilon}$-spaced specification $S$ is $\varepsilon$-shadowed by a point of $X$.

The next result is stated without proof in [Sigmund 1974]. We provide a proof for completeness.

Lemma 3.3. If $(X, f)$ has the specification property and $(Y, g)$ is a factor of $(X, f)$, then $(Y, g)$ has the specification property.

Proof. Fix $\varepsilon>0$ and let $d_{X}$ and $d_{Y}$ denote metrics for $X$ and $Y$, respectively. Let $\varepsilon^{\prime}>0$ such that if $d_{X}\left(x_{1}, x_{2}\right)<\varepsilon^{\prime}$, then

$$
d_{Y}\left(h\left(x_{1}\right), h\left(x_{2}\right)\right)<\varepsilon,
$$

where $x_{1}, x_{2} \in X$. Such an $\varepsilon^{\prime}>0$ can always be chosen since $h$ is continuous. Fix $M_{\varepsilon^{\prime}} \in \mathbb{N}$ such that any $M_{\varepsilon^{\prime}}$-spaced specification is $\varepsilon^{\prime}$-shadowed by a point of $X$ and let $M_{\varepsilon}=M_{\varepsilon^{\prime}}$. 
Let $S=(\tau, P)$ be an $M_{\varepsilon}$-spaced specification in $(Y, g)$ where $\tau=\left\{I_{1}, \ldots, I_{m}\right\}$ is a collection of $M_{\varepsilon}$-spaced intervals. Let

$$
B=\left\{y_{1}, y_{2}, \ldots, y_{m}\right\} \subset Y,
$$

where $y_{i}=P\left(a_{i}\right)$ for all $1 \leq i \leq m$.

Fix $A=\left\{x_{1}, \ldots, x_{m}\right\} \subset X$ such that $h$ restricted to $A$ is a bijection onto $B$ and $h\left(x_{i}\right)=y_{i}$ for $1 \leq i \leq m$. The orbit segment for $x_{i}$ in $I_{i}$ is given by

$$
\left\{f^{a_{i}}\left(x_{i}\right), \ldots, f^{b_{i}}\left(x_{i}\right)\right\} \text { for } 1 \leq i \leq m .
$$

Define $P_{X}: \bigcup_{i=1}^{m} I_{i} \rightarrow X$ such that $P\left(a_{i}\right)=x_{i}$ for all $i$ such that $1 \leq i \leq m$.

Now, given that $(X, f)$ has the specification property, we know there exists an $\varepsilon^{\prime}$-shadowing point $x$ for the specification $\left(\tau, P_{X}\right)$ and $h(x) \in Y$. Furthermore,

$$
h\left(f^{a_{i}}\left(x_{i}\right)\right)=g^{a_{i}}\left(y_{i}\right)
$$

since $h$ is a semiconjugacy. Hence, $d(h(x), h(P(n)))<\varepsilon$ for all $n \in \bigcup_{i=1}^{m} I_{i}$ and $h(x)$ is an $\varepsilon$-shadowing point for the specification $S$.

Theorem 3.4 [Bowen 1974]. Let $X$ be a compact metric space and $f$ be an expansive homeomorphism with the specification property. Then $f$ is intrinsically ergodic.

Weiss [1973] showed that a mixing subshift of finite type has the specification property. Since subshifts of finite type are expansive, we know from Theorem 3.4 that a topologically mixing subshift of finite type is intrinsically ergodic.

From Lemma 3.3 we know that a factor of a mixing subshift of finite type is intrinsically ergodic.

Corollary 3.5. Any topologically mixing finitely presented system is intrinsically ergodic.

Proof. Let $(X, f)$ be a topologically mixing finitely presented system. To prove the corollary we show there is a topologically mixing subshift of finite type that is an extension of $(X, f)$. Let $\mathscr{R}$ be a Markov partition for $(X, f)$ and $A$ be the adjacency matrix associated with $\mathscr{R}$. Let $R_{i}$ and $R_{j}$ be rectangles in $\mathscr{R}$. Since $(X, f)$ is topologically mixing and the rectangles are proper, we know there exists some $N_{i j} \in \mathbb{N}$ such that $f^{n}\left(\stackrel{\circ}{R}_{i}\right) \cap \stackrel{\circ}{R}_{j} \neq \varnothing$ for all $n \geq N_{i j}$. Using arguments as in the proof of Theorem 1.1, we know that $a_{i j}^{n}>0$ for all $n \geq N_{i j}$. Define $N=\max \left(N_{i j}\right)$. Then $A^{n}$ is positive for all $n \geq N$ and the subshift of finite type associated with the Markov partition $\mathscr{R}$ is topologically mixing.

Proof of Corollary 1.2. Let $f \in \operatorname{Diff}(M)$ for some manifold $M$, let $\Lambda$ be a topologically mixing hyperbolic set for $f$, and $V$ be a neighborhood of $\Lambda$. From Theorem 1.1 we know that there exists a topologically mixing hyperbolic set $\tilde{\Lambda}$ contained 
in $V$ and containing $\Lambda$ with a Markov partition. Therefore, $\tilde{\Lambda}$ is finitely presented and from Corollary 3.5 we know that $\tilde{\Lambda}$ is intrinsically ergodic.

\section{References}

[Adler and Weiss 1967] R. L. Adler and B. Weiss, "Entropy, a complete metric invariant for automorphisms of the torus", Proc. Nat. Acad. Sci. U.S.A. 57 (1967), 1573-1576. MR 35 \#3031 Zbl 0177.08002

[Bowen 1974] R. Bowen, "Some systems with unique equilibrium states", Math. Systems Theory 8:3 (1974), 193-202. MR 53 \#3257 Zbl 0299.54031

[Brin and Stuck 2002] M. Brin and G. Stuck, Introduction to dynamical systems, Cambridge University Press, Cambridge, 2002. MR 2003m:37001 Zbl 01849967

[Fisher 2006] T. Fisher, "Hyperbolic sets that are not locally maximal", Ergodic Theory Dynam. Systems 26:5 (2006), 1491-1509. MR 2008a:37031 Zbl 1122.37022

[Fried 1987] D. Fried, "Finitely presented dynamical systems", Ergodic Theory Dynam. Systems 7:4 (1987), 489-507. MR 89h:58157 Zbl 0652.54028

[Katok and Hasselblatt 1995] A. Katok and B. Hasselblatt, Introduction to the modern theory of dynamical systems, Encyclopedia of Mathematics and its Applications 54, Cambridge University Press, Cambridge, 1995. MR 96c:58055 Zbl 0878.58020

[Robinson 1999] C. Robinson, Dynamical systems: stability, symbolic dynamics, and chaos, 2nd ed., CRC Press, Boca Raton, FL, 1999. MR 2001k:37003 Zbl 0914.58021

[Sigmund 1974] K. Sigmund, "On dynamical systems with the specification property", Trans. Amer. Math. Soc. 190 (1974), 285-299. MR 50 \#4898 Zbl 0286.28010

[Weiss 1973] B. Weiss, "Subshifts of finite type and sofic systems", Monatsh. Math. 77 (1973), 462-474. MR 49 \#5308 Zbl 0285.28021

Received: 2009-01-13 Revised: 2009-09-01 Accepted: 2009-10-28

tfisher@math.byu.edu

Department of Mathematics, Brigham Young University, Provo, UT 84602, United States

http://math.byu.edu/ tfisher/

himal46@gmail.com

Department of Mathematics, Brigham Young University,

Provo, UT 84602, United States 